\title{
Observation and Identification of Metastable Excited States in Ultrafast Laser-lonized Pyridine
}

\author{
David B. Foote, Timothy D. Scarborough, Cornelis J. G. J. Uiterwaal
}

Department of Physics and Astronomy, University of Nebraska-Lincoln, Lincoln, NE 68588-0299, USA

\begin{abstract}
We report on the fragmentation of ionized pyridine $\left(\mathrm{C}_{5} \mathrm{H}_{5} \mathrm{~N}\right)$ molecules by focused $50 \mathrm{fs}, 800 \mathrm{~nm}$ laser pulses. Such ionization produces several metastable ionic states that fragment within the field-free drift region of a reflectron-type time of flight mass spectrometer, with one particular metastable dissociation being the leading fragmentation process. Because the time of flight is no longer dependent in a simple way on the mass of the ion, the metastable decay is manifested as an unfocused peak on the mass spectrum that appears at a time of flight not corresponding to an integer mass. A previously-developed method is used to identify the precursor and final masses of these ions. The metastable process that creates the most prevalent peak is shown to be $\mathrm{C}_{5} \mathrm{H}_{5} \mathrm{~N}^{+} \rightarrow \mathrm{C}_{4} \mathrm{H}_{4}{ }^{+}+\mathrm{HCN}$. Simulations confirm this result and place restrictions on the processes for several other observed metastable reactions.
\end{abstract}

Key words: Metastable, Ultrafast, Pyridine, Intense fields, Fragmentation, Ionization, Time of flight, Reflectron

\section{Introduction}

$\mathrm{T}$ he identification of metastable ionic species in organic molecules has been a topic of some scientific interest in the past several decades, particularly as a tool for analyzing gaseous samples $[1,2]$. These long-living states of ionized molecules were readily studied in conjunction with laser ionization processes that span the picosecond-to-microsecond range, particularly using $(1+1)$ resonance-enhanced multiphoton ionization (REMPI) [3-6]. These efforts have centered around developing an alternate setup of a reflectron-type time-of-flight (TOF) mass spectrometer to identify metastable effects [7-12]. The analysis of these species in a reflectron TOF spectrometer, even in the acceleration region [13], is thorough and well-documented, as is similar

Electronic supplementary material The online version of this article (doi:10.1007/s13361-012-0346-6) contains supplementary material, which is available to authorized users.

Correspondence to: David B. Foote; e-mail: dbfoote@huskers.unl.edu analytical work involving other configurations of mass spectrometers [14-16]. While similar effects have also been observed in excited ionic states of molecules ionized on the ultrafast time scale [17, 18], little experimental work has been done in modeling or categorizing such metastable species, though the electronic spectra [19] and VUV ionization [20] have been well-documented.

The lack of continuation of this research into the femtosecond regime is unfortunate, because faster time scale (and thus higher intensity) processes offer a much greater flexibility in the investigation of metastables. For example, nanosecond laser pulses with small electric fields must be tuned to match a resonance to take advantage of REMPI ionization; ultrashort pulses have a greater frequency bandwidth, as a direct consequence of the time-energy uncertainty relation, which makes the resonance condition more flexible. In addition, high-field lasers can force a resonance in a given molecule [21] through a so-called "dynamic resonance" [22], allowing identical pulses to access resonances in many different molecules. Because of 
this, metastable features previously hidden can be studied through femtosecond ionization.

In a typical molecular fragmentation event, decay occurs quickly such that the travel as the molecular parent species is negligible, and the TOF is determined entirely by the properties (mass and charge) of the daughter ion. In the event of a very long-living ionic species, which survives into the drift region of a mass spectrometer, one must consider the dependence on the parent ion's drift velocity and the daughter ion's mass, along with distributions of breakup distance and kinetic energy release (KER). Such a slow decay process is indicated by a broad, symmetric feature in the TOF spectrum that lands at a total TOF corresponding to no ratio of integer mass and charge. The mass difference will place the final observed peak at a total TOF greater than that of the daughter ion, but less than that of the parent ion. The KER will cause a broadening of this intermediary peak that cannot be resolved by the reflectron. We call this sort of dissociation metastable: in the context of the present experiment, a metastable process is one with a lifetime greater than $\sim 80 \mathrm{~ns}$ (the time to enter the field-free drift region).

Figure 1 shows an example of the most prominent broad feature in the TOF spectrum of pyridine $\left(\mathrm{C}_{5} \mathrm{H}_{5} \mathrm{~N}\right)$. We have observed similar peaks in a number of other similarlyionized molecules: 1,2-diazine, 1,3-diazine, 1,4-diazine (all $\left.\mathrm{C}_{4} \mathrm{H}_{4} \mathrm{~N}_{2}\right)$, phenol $\left(\mathrm{C}_{6} \mathrm{H}_{5} \mathrm{OH}\right)$, aniline $\left(\mathrm{C}_{6} \mathrm{H}_{5} \mathrm{NH}_{3}\right)$, toluene $\left(\mathrm{C}_{6} \mathrm{H}_{5} \mathrm{CH}_{3}\right)$, fluorobenzene $\left(\mathrm{C}_{6} \mathrm{H}_{5} \mathrm{~F}\right)$, bromobenzene $\left(\mathrm{C}_{6} \mathrm{H}_{5} \mathrm{Br}\right)$, and iodobenzene $\left(\mathrm{C}_{6} \mathrm{H}_{5} \mathrm{I}\right)$. Unlike pyridine, however, the broad features in each of these molecules are insubstantial compared with their molecular parent peaks. Even more intriguing is the systematic nature that these unfocused peaks have; for example, the three diazines all display a feature similar to that in pyridine, landing at the same TOF.

What makes pyridine a prime candidate to investigate is its relative propensity to undergo metastable fragmentation. The broad peak contains a comparable number of counts to the parent ion peak, which remains the dominant feature. As Figure 2 shows, the total ion yield as a function of intensity for the broad peak closely follows that of the molecular parent at high intensities, suggesting that the metastable fragmentation originates from the parent ion. The two curves do deviate at low intensities; this could be indicative of an intensity-dependent population of a metastable excited ionic state. However, at present, no solid conclusions can be drawn in this regard. Pyridine was ionized with focused, high-intensity, $50 \mathrm{fs}$ laser pulses at $800 \mathrm{~nm}$, with $(3+3)$ REMPI being the dominant ionization mechanism [23]. The data presented in Figure 2 differs from the REMPI experiment in that Figure 2 represents only 2-dimensional spatial resolution; the REMPI signatures are not visible here, but this more accurately describes the comparative yields of the parent and metastable ions.

We assume that these broad peaks arise from the metastable dissociation of an ion (not necessarily the singly-ionized molecular parent) in the drift region of the TOF. Part and parcel with this assumption are four unknowns - the parent ion mass, the daughter ion mass, the dissociation lifetime, and the KER distribution. A

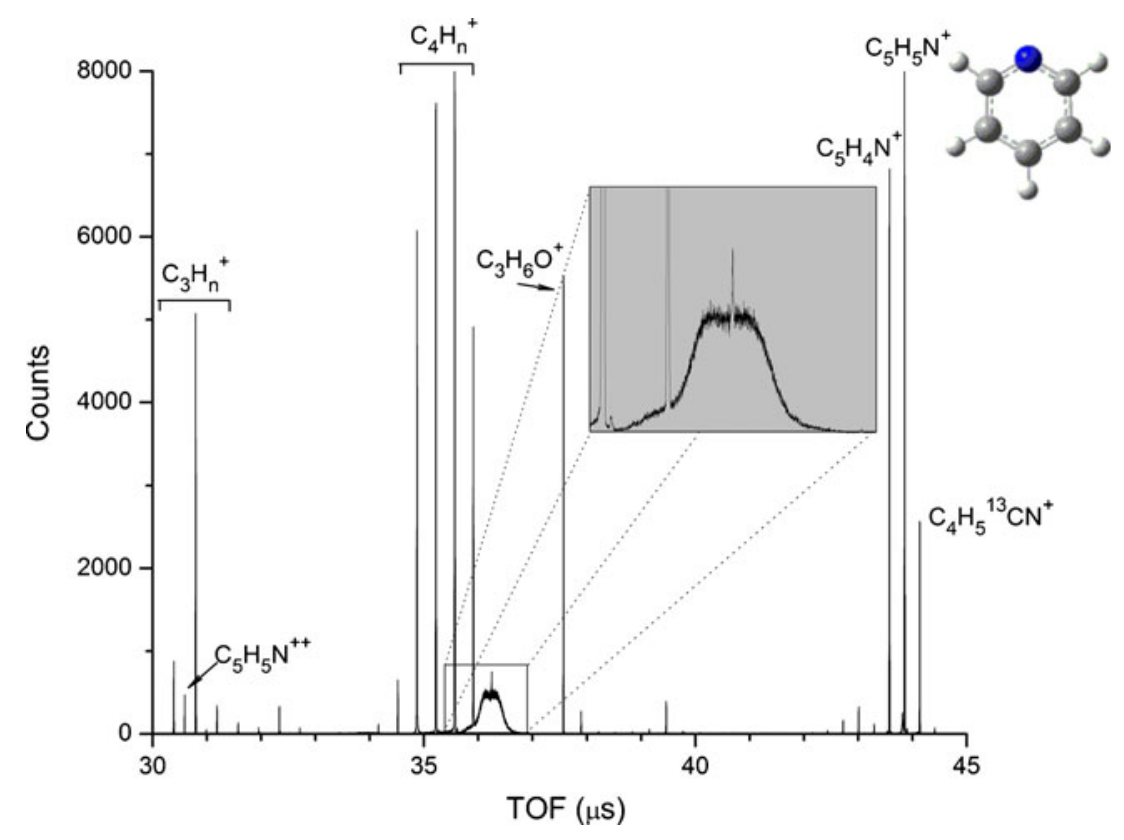

Figure 1. The focused TOF spectrum for pyridine $\left(\mathrm{C}_{5} \mathrm{H}_{5} \mathrm{~N}\right)$. While all other peaks are confined to temporal regions less than 10 ns wide, there is an unmistakable broad peak ( 500 ns in full-width at half-maximum) landing at approximately $36 \mu$ s. Some surrounding prompt ionic fragments are included for reference. The coincident focused peak, appearing on top of the unfocused peak, is due to a normally-produced ionic fragment, and is not related to the metastable feature 


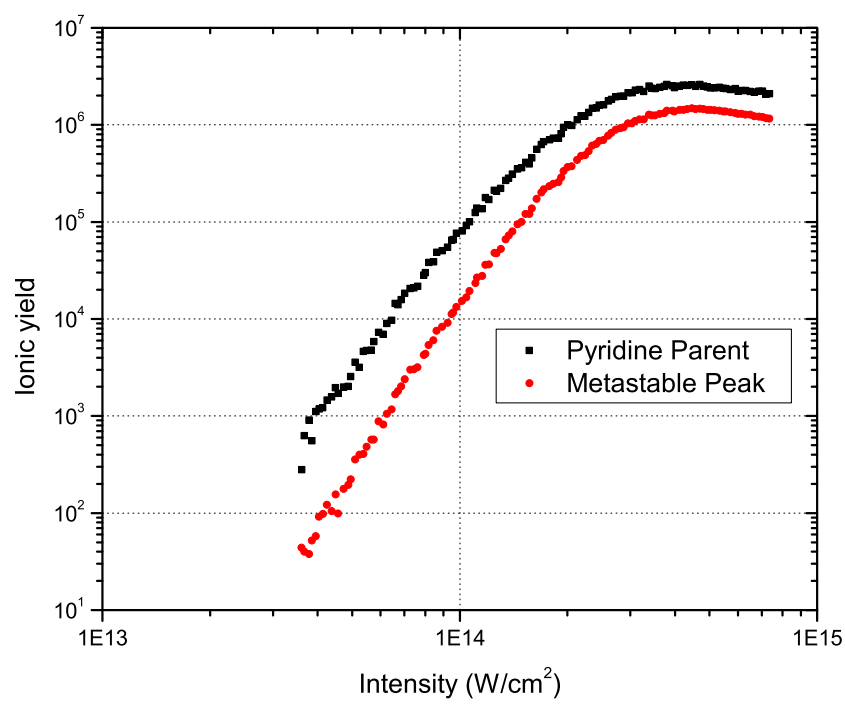

Figure 2. The total ionic yield as a function of laser intensity for singly-ionized pyridine (black squares) and the metastable state (red circles). The correspondence of total yield at high intensity suggests that this metastable feature arises from the ionized parent. At low intensities, poor statistics inhibit rigorous analysis of the data, but the metastable state clearly falls further under the parent ion. It is a hypothesis that the excited ionic state responsible for the metastable dissociations is only substantially populated at high intensities

method for obtaining information on such metastable dissociations in TOF mass spectrometers was presented in reference [3]. In the present work, this method of tuning reflectron voltages to energy-select ions in the TOF is employed to identify dissociative products and propose metastable pathways for pyridine. Simulating the proposed dissociation is shown to produce similar results to those obtained experimentally. These methods still place no restrictions upon the lifetime of the decay, other than that it must be in a range such that most of the dissociations happen within the field-free drift region of the TOF.

\section{Experimental}

\section{Materials and Equipment}

All chemicals were purchased from Sigma-Aldrich (St. Louis, MO, USA) and used as purchased. Pyridine was purchased at $99.8 \%$ purity; all other chemicals were purchased at no less than $98 \%$ purity, with the exception of phenol, which was purchased in aqueous solution and dehydrated before use.

A Ti:sapphire amplified laser beam, which produces 800 $\mathrm{nm}$ pulses of $50 \mathrm{fs}$ duration at 1000 pulses per $\mathrm{s}$ was used. This corresponds to approximately $1.55 \mathrm{eV}$ per photon. With $\sim 2 \mathrm{~mJ}$ per pulse, the amplified beam can create intensities of greater than $10^{15} \mathrm{~W} / \mathrm{cm}^{2}$ when focused. A half-wave plate inside the amplifier allowed variable attenuation of the beam from $\sim 10^{13} \mathrm{~W} / \mathrm{cm}^{2}$ to the maximum intensity. For the purposes of this experiment, between $80 \mathrm{~mW}$ and $1.7 \mathrm{~W}$ of total power was used.

\section{Time-of-Flight Mass Spectrometer}

Figure 3 shows the experimental setup of the TOF mass spectrometer with relevant distances and voltages identified. The system is a standard reflectron-type TOF, and as such the spacings and potentials of the three meshes that constitute the reflecting region importantly affect the kinematics of the ion. In a typical experiment in our spectrometer, the middle mesh potential $V_{2}$ is held at approximately $800 \mathrm{~V}$, while the back mesh potential $V_{3}$ is held close to $1050 \mathrm{~V}$. The distances $\mathrm{d}_{2}$ and $\mathrm{d}_{3}$ are 14.64 and $6.16 \mathrm{~cm}$, respectively, so a larger retarding force is applied to the ions in the first deceleration region. The laser pulses are focused into a low-pressure chamber which contains the target vapor at a pressure of $\sim 10^{-7}$ mbar. This pressure was chosen to maximize total counts without introducing the distortions in data that excessive count rates and pressure effects can create. The molecules that are ionized in the focus are accelerated away from the repeller plate (held at $V_{r}=1530 \mathrm{~V}$ ) toward the acceptance slit (dimensions $15 \times$ $500 \mu \mathrm{m}$ ), which is grounded. It is accelerated to a velocity such that its kinetic energy is

$$
T=\frac{d_{0}}{d_{r}} q V_{r}
$$

where $d_{r}$ is the distance from the repeller plate to the slit, and $d_{0}$ is the distance from the point at which the ion was created to the slit. The acceleration of the ion in this region is

$$
a_{r}=\frac{q V_{r}}{d_{r} m_{i}}
$$

and the ion's velocity at the moment it enters the drift region of the TOF (where $V=0$ ) is

$$
v_{a}=\left(\frac{2 d_{0} q V_{r}}{d_{r} m_{i}}\right)^{\frac{1}{2}}
$$

Here, $m_{i}$ is the initial mass of the ion. After entering into the drift chamber, the ion breaks apart into (at least) two fragments via a metastable dissociation at some distance $d_{e}$ (with the subscript denoting some "explosion" into fragments). Assuming the initial ion carries unit charge, only one of the fragments will likely be charged after the dissociation. This charged fragment will continue traveling at the predissociation velocity (with some additional, comparatively small component due to KER in the dissociation), but have an altered, smaller mass. Therefore, the kinetic energy will be less than that of the parent. Because of this reduced kinetic energy, the fragments are turned around in the reflecting section of the TOF (between grids 1 and 3 as 


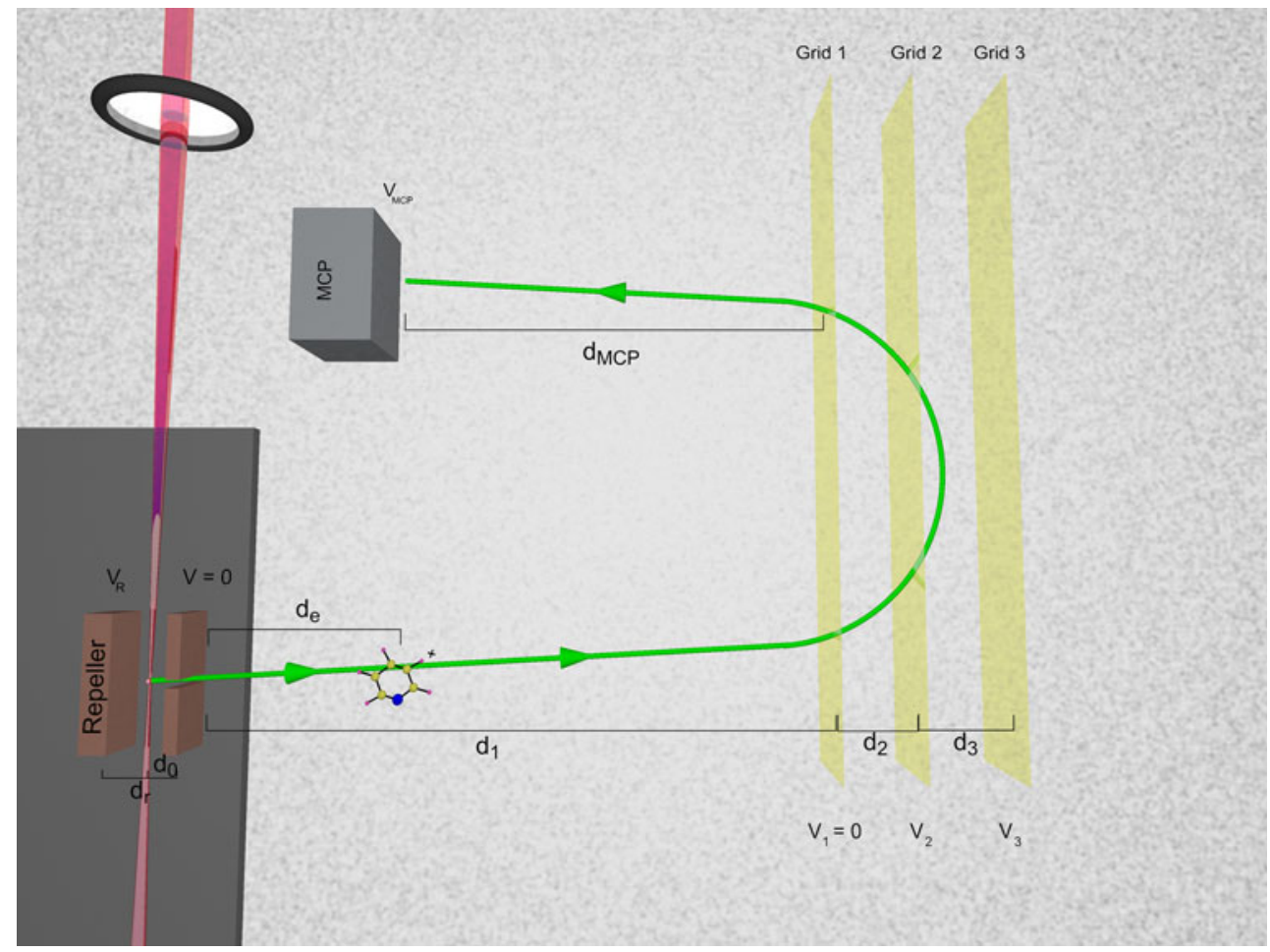

Figure 3. TOF schematic. The ionic parent is created at a distance $d_{0}$ from the acceptance slit (grounded). The acceleration is due to the field caused by $V_{R}$ over a distance $\mathrm{d}_{\mathrm{r}}$. For metastable ions, it is assumed here that the dissociation occurs somewhere between the repeller and grid 1 (the field-free drift region). The distance at which this occurs is labeled $d_{e}$ for modeling purposes. Upon reaching the positive-voltage grids, the ion is decelerated and reflected back toward a multichannel plate, where it is detected

labeled in Figure 3) differently than the parent ions and as such do not land at a ratio of integer $m$ and $z$ values in the mass spectrum. The exact formula for the TOF for the metastable fragments is stated in the Supplementary Material, and is used for modeling purposes. Also, if the final mass is sufficiently small, the daughter ions will be turned around in the first deceleration region (between meshes 1 and 2), where there is a large potential difference over a small distance. When this happens, the TOF becomes a single-stage reflectron device for these ions, which is why it is unable to focus these metastable ions in time.

Let us state the assumptions taken in order to reach this point. First, it is assumed that the ion breaks apart in the first drift region $\left(d_{e}<d_{1}\right)$. Second, the angle at which the additional velocity is added to the trajectory must be sufficiently small that the ion still reaches the multichannel plate $(3 \mathrm{~cm}$ diameter). This acceptance angle will depend on the total TOF of the fragment. Third, it is assumed that the ion has sufficiently small kinetic energy to be turned around in the deceleration region.

The first assumption is taken as a hypothesis for the experiment. The first and second assumptions are accounted for in the code used for modeling (see Supplementary Material for details). For the third assumption, to avoid being transmitted through grid 3 (and thus disappearing from the mass spectrum), the kinetic energy must be less than some cutoff voltage, which is determined by the potential of grid 3. Mathematically, we have for ions with kinetic energy

$$
T_{e}=\frac{1}{2} m_{f} v_{a}^{2}=\frac{d_{0} m_{f}}{d_{r} m_{i}} q V_{r}
$$

For ions to be detected, $q V_{3}$ must be greater than this kinetic energy. In fact, this condition can be used to identify the final masses of the daughter ions, as described by reference [3].

By decreasing $V_{3}$ until the daughter ions disappear from the TOF spectrum and using the relation

$$
V_{3}=\left(\frac{d_{0}}{d_{r}}\right)\left(\frac{m_{f}}{m_{i}}\right) V_{r}
$$

from equating the two expressions for energy, one can solve for the ratio $\frac{m_{f}}{m_{i}}$. In the present work we used this approach to investigate the nature of several of the metastable peaks in the pyridine mass spectrum.

The particular tuning of the middle voltage $V_{2}$ in a reflectron TOF setup allows a spatial resolution of the laser focus in the final mass spectrum, as described elsewhere [24], and indeed this tuning of the spectrometer was where the broad peaks were first observed in our mass spectra. A retuning of this voltage allows this effect to be negated, 
creating a flat TOF dispersion curve throughout the interaction region ("flat" here means temporally focused; the TOF is independent of the initial location of the ion in the focus). It is important to rule out the spatial resolution of reference [24] for the monotonically increasing case as being some unaccounted source of the spreading of the observed features. As such, data was taken on both time-focused and spatially-resolved tunings of the spectrometer to observe the effect of changing the voltage on the structure of each peak. As expected, the form of the wide feature did not change for different tunings.

\section{Results and Discussion}

\section{Experimental Results}

The cutoff voltage $V_{3}$ was successively lowered until all of the stable ions produced in the laser focus could no longer be reflected and thus escaped detection. Measuring the extinction of the stable peaks as a function of $V_{3}$ allowed the measurement of the initial position of the ions in the TOF chamber (the position of the focus) $d_{0}=1.993 \pm 0.005 \mathrm{~mm}$. As the cutoff potential was continually lowered, it became apparent that the main metastable peak observed in the original mass spectrum was in fact three separate features, closely spaced, as shown in Figure 4. By keeping the middle potential $V_{2}$ proportional to $V_{3}$ as it was when the normal ions were tuned, the metastable peaks were similarly brought into sharp focus. Obtaining results on the KER distribution from this effect is described later in this section. The three peaks will be henceforth referred to by their numbering in Figure 5.

The extinction voltage for each peak is the voltage at which the ion yield per unit time for that peak is exactly $50 \%$ of the full ion yield. These extinction voltages, and the corresponding $\frac{m_{f}}{m_{i}}$ ratio as determined from Equation 5, are shown in Table 1. With the assumption that the parent ion lies somewhere in the parent or parent-minus-hydrogen group $\left(\frac{m}{z}=78,79,80\right)$, the daughter ion falls somewhere in the $\mathrm{C}_{4} \mathrm{H}_{n}$ group. Specifically, if for (2), the dominant metastable feature, $m_{i}=79 \mathrm{Da}$, then $m_{f} \cong 52 \mathrm{Da}$ suggests the decay $\mathrm{C}_{5} \mathrm{H}_{5} \mathrm{~N}^{+} \rightarrow \mathrm{C}_{4} \mathrm{H}_{4}^{+}+\mathrm{HCN}$. This particular decay channel has been previously documented in pyridine and pyrazine $[25,26]$.

While it seems reasonable to assume that (2) comes from the parent ion given its abundance, (1) and (3) become more ambiguous. Either could come from a heavy parent molecular ion, $\mathrm{C}_{4} \mathrm{H}_{5} \mathrm{~N}\left({ }^{13} \mathrm{C}\right)^{+}$, or potentially $\mathrm{C}_{5} \mathrm{H}_{4} \mathrm{~N}^{+}$, which is photochemically similar to the ionic parent. Conclusive results from the experimentally-determined mass ratios are not attainable, but simulations can help to determine the parent and final ion states, as is done in the Supplementary Material. (1) and (3) were shown by modeling to have likely come from $m_{f} \rightarrow m_{i}: 80 \rightarrow 53 \mathrm{Da}$ and $m_{f} \rightarrow m_{i}: 78 \rightarrow 51 \mathrm{Da}$, respectively. The conclusion for (1) can be further reinforced by looking at the ratio of total counts in (1) and (2). By

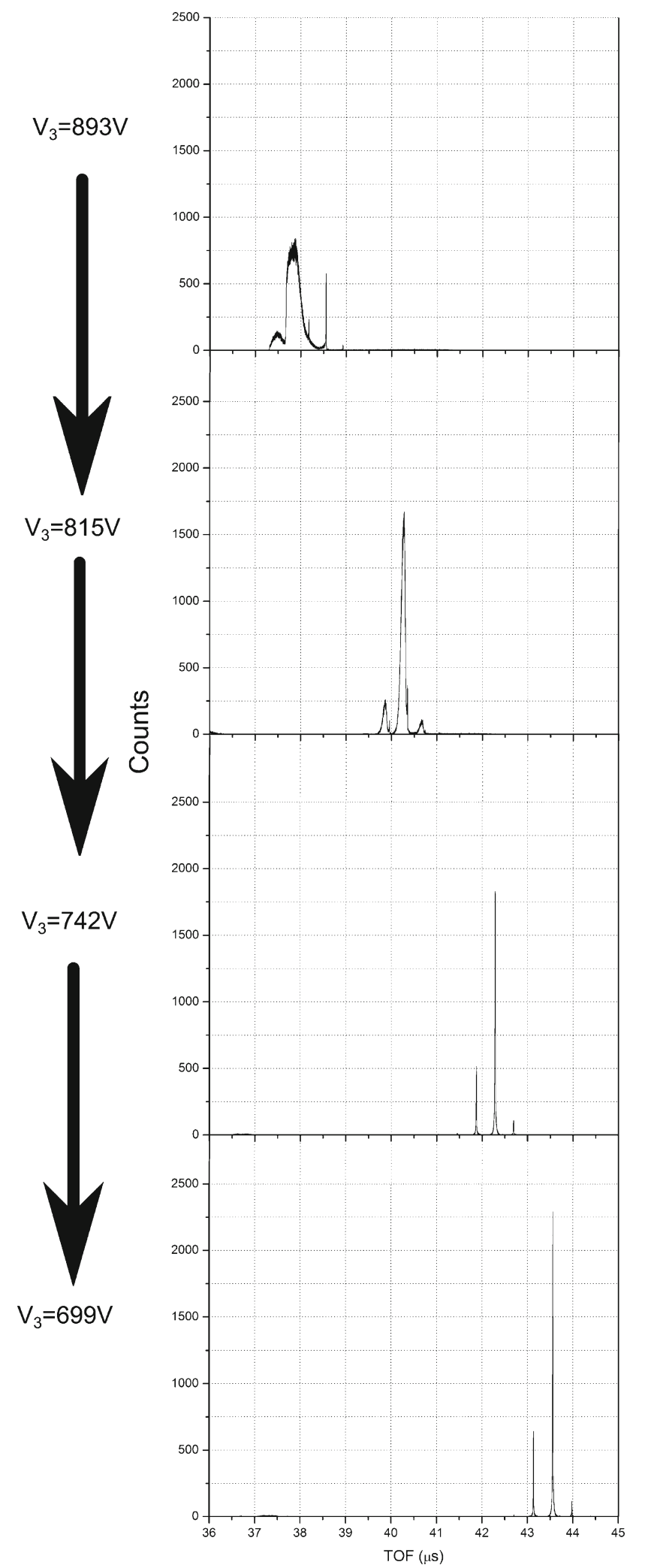

Figure 4. Metastable peak evolution as a function of reflecting voltage. As the potential $V_{3}$ is decreased (with $V_{2}$ decreasing in proportion) from top to bottom, the single broad peak evolves into three distinct (tuned) peaks coming from three separate processes, and also migrates to a longer flight time 


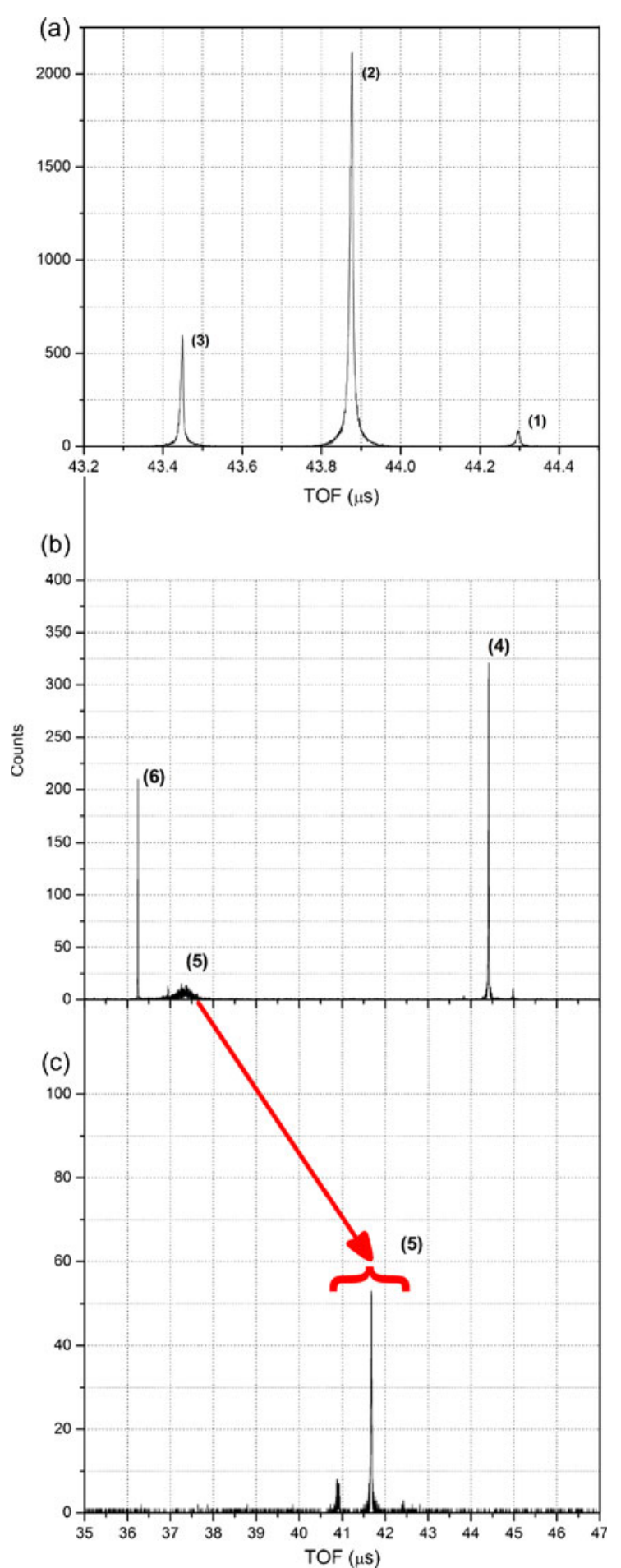

Figure 5. Labeling schemes. (a) The labeling scheme for the peaks (1), (2), and (3); for this spectrum, $V_{3}=689 \mathrm{~V}$, which is a potential just above the point at which these peaks start to disappear. All three exhibit a broadening at the tail, likely indicative of a kinetic energy release during dissociation. (b) and (c) Labeling scheme and decreasing voltage progression of (4), (5), and (6). Between (b) $V_{3}=504$ and (c) $V_{3}=410 \mathrm{~V}$, (4) and (6) disappear, while (5) is brought into focus and moves substantially to the right. The significantly different behavior of (5) suggests a different mode of origin, possibly from a doubly-ionized parent considering the natural abundances of isotopes of carbon and nitrogen, one can determine about $5.92 \%$ of pyridine molecules should be one Da heavier. Direct measurement of the counts in (1) and (2) at $V_{\mathrm{a}}=776$ yields the ratio (1)/ $[(\mathbf{2})+(\mathbf{1})]=0.060$.

As these three features disappear ( $V_{3}$ drops below $\sim 670$ $\mathrm{V})$, three more diffuse peaks begin to become recognizable on the mass spectrum. As one continues to lower the cutoff potential, these peaks become similarly focused. The decay voltages and corresponding $\frac{m_{f}}{m_{i}}$ ratios for these three peaks [similarly labeled (4), (5), and (6)] are determined and also summarized in Table 1.

The $\frac{m_{f}}{m_{i}}$ ratios suggest that (4) and (6) represent a $\mathrm{C}_{3} \mathrm{H}_{\mathrm{n}}$ fragment after decaying from the molecular parent; (5) would have to represent the molecular parent decaying to a fragment of $m_{f}=28$ under this assumption, which is the mass of no easily imaginable fragment of pyridine. Furthermore, if the same process occurred for (5) as for (4) and (6), one would expect this peak to lie at a lower TOF than the other two, as it has a substantially smaller final mass and is therefore turned around much more quickly in the reflecting portion of the mass spectrometer. In reality, it is observed between (4) and (6) (see Figure 5), which also suggests some entirely different metastable process involved in the creation of this fragment. This ion is perhaps a product of some other ionic fragment, or perhaps it decays from a multiply-ionized parent. The latter seems likely as it appears to resolve into a triplet peak shortly before extinction, similar to the (1), (2), (3) triplet. Our experiment is capable of producing multiply-charged ions [23], and the existence of the prompt multiply-charged parent ion is shown in Figure 1. Mass calibration of the TOF data in Figure 1 results in $m / z=39.5$ for this peak.

In order to make a statement about the KER distribution, consider first the kinetic energy distribution of ions that are created in the laser focus and do not decay. The kinetic energy of these ions is determined entirely (up to thermal effects) by where the ion was created in the focus. Ions at different positions in the focus experience the electric field of the acceleration region of the TOF for differing distances, creating some spread of kinetic energies. By measuring the extinction curve for the molecular parent ion, one can obtain the kinetic energy distribution of the ion, since the extinction curve statistically represents the continuous distribution function for kinetic energy. This kinetic energy distribution was found to have a $\frac{1}{e^{2}}$ width of about $10.1 \mathrm{eV}$. The total kinetic energy distributions of the six metastable peaks are determined similarly, and the process and results are summarized in Figure 6 and Table 1.

The fragments produced by metastable decay will retain the kinetic energy distribution from their parent ion in the focus, but likely have some additional kinetic energy due to the exothermic bond breaking during decay. As Table 1 shows, (1), (2), and (3) do indeed have somewhat wider kinetic energy peaks than the molecular parent. However, (4), (5), and (6) have noticeably smaller widths than the 
Table 1. Extinction Voltages for the Six Observed Metastable Peaks. From this and Equation 5, the Mass Ratio can be Calculated. This Mass Ratio is used to Claim Dissociative Pathways for each of the Metastable Peaks. These Pathways are only taken as a Hypothesis, and (4) and (6) are Shown to not be the Result of $79 \rightarrow 39$, 38 When One Considers the KE Distribution; (5) is Speculative, and Merely States that the Peak could likely Result from the Doubly-Charged Molecular Parent Decaying into some $\mathrm{C}_{4} \mathrm{H}_{\mathrm{n}}$ Fragment (Possibly Multiply-Charged as well). The Kinetic Energy Distribution Width for the Metastable Peaks is Assumed to have a Component Due to Kinetic Energy Release, so the Width should be at least as Wide as the Parent

\begin{tabular}{|c|c|c|c|c|c|}
\hline Peak & $\mathrm{V}_{\mathrm{E}}(\mathrm{V})$ & $\mathrm{m}_{\mathrm{f}} / \mathrm{m}_{\mathrm{i}}$ & Likely $\mathrm{m}_{\mathrm{f}} \rightarrow \mathrm{m}_{\mathrm{i}}(\mathrm{Da})$ & Hypothesized dissociation scheme & KE dist. width (eV) \\
\hline (1) & $679.9 \pm 0.719$ & 0.669 & $80 \rightarrow 53$ & $\mathrm{C}_{4} \mathrm{H}_{5}{ }^{13} \mathrm{CN}^{+} \rightarrow \mathrm{C}_{3} \mathrm{H}_{4}{ }^{13} \mathrm{C}^{+}+\mathrm{HCN}$ & 10.2 \\
\hline (2) & $673.1 \pm 0.793$ & 0.662 & $79 \rightarrow 52$ & $\mathrm{C}_{5} \mathrm{H}_{5} \mathrm{~N}^{+} \rightarrow \mathrm{C}_{4} \mathrm{H}_{4}^{+}+\mathrm{HCN}$ & 11.4 \\
\hline (3) & $670.4 \pm 0.311$ & 0.660 & $78 \rightarrow 51$ & $\mathrm{C}_{5} \mathrm{H}_{4}^{+} \rightarrow \mathrm{C}_{4} \mathrm{H}_{3}^{+}+\mathrm{HCN}$ & 14.2 \\
\hline (4) & $492.9 \pm 0.585$ & 0.490 & $79 \rightarrow 39$ & not identified & 6 \\
\hline (5) & $358.9 \pm 0.773$ & 0.357 & $79 \rightarrow 52$ & $\mathrm{C}_{5} \mathrm{H}_{5} \mathrm{~N}^{++} \rightarrow \mathrm{C}_{4} \mathrm{H}_{\mathrm{n}}^{+(+)}+\mathrm{H}_{(5-\mathrm{n})} \mathrm{CN}$ & 7.5 \\
\hline (6) & $488.7 \pm 0.374$ & 0.486 & $79 \rightarrow 38$ & Not identified & 7.1 \\
\hline Parent & $1013 \pm 1.11$ & N/A & N/A & N/A & 10.1 \\
\hline
\end{tabular}

parent ion. This suggests that all three peaks and not just (5), as proposed earlier, decay from some ion besides the singlyionized parent. The widths for these precursor ions could be smaller than that of the molecular parent because the region of the laser focus in which light is intense enough to produce these ions (the interaction volume) can be smaller for fragments and multiply-ionized states than it is for singlyionized pyridine. As a great number of fragments and multiply-ionized states will require a higher laser intensity to be produced (and thus be limited to a smaller interaction volume), such a conclusion fails to isolate a single new candidate for the metastable parent.

This conclusion is reinforced by simulation, details of which can be found in the Supplementary Material. In short, the simulation reconstructs the kinematics of an ion that dissociates in the drift region of the TOF chamber and records the total TOF for a large number of instances. The resulting histogram replicates the broad features observed. By simulating a decay of the molecular parent, heavy parent, or deprotonated parent ion into $\mathrm{C}_{3} \mathrm{H}_{\mathrm{n}}{ }^{+}$, as was assumed to be the process that led to (6) with the same potentials used in the experiment, one comes up with a total TOF between 43 and $46 \mu$ s, which does not match the $36.25 \mu$ s TOF observed in the experimental data. Clearly, this trio of metastable peaks, or at the very least (5) and (6), come from a different parent ion than the singly-charged molecular parent.

\section{Conclusion}

Metastable ions are known not to be unique to pyridine; in fact, the appearance of metastables in TOF mass spectra seems to be the norm rather than the exception in aromatic systems. This is evidenced by the number of aromatic molecules in which we have observed similar metastable features. As our data on the six observed metastable states have shown, pyridine specifically presents a wealth of informative results on metastable processes, some of which are uniquely accessible through femtosecond ionization. By deliberately detuning the potentials applied to the reflecting meshes of a reflectron TOF mass spectrometer, the specific fragmentation pathways of three

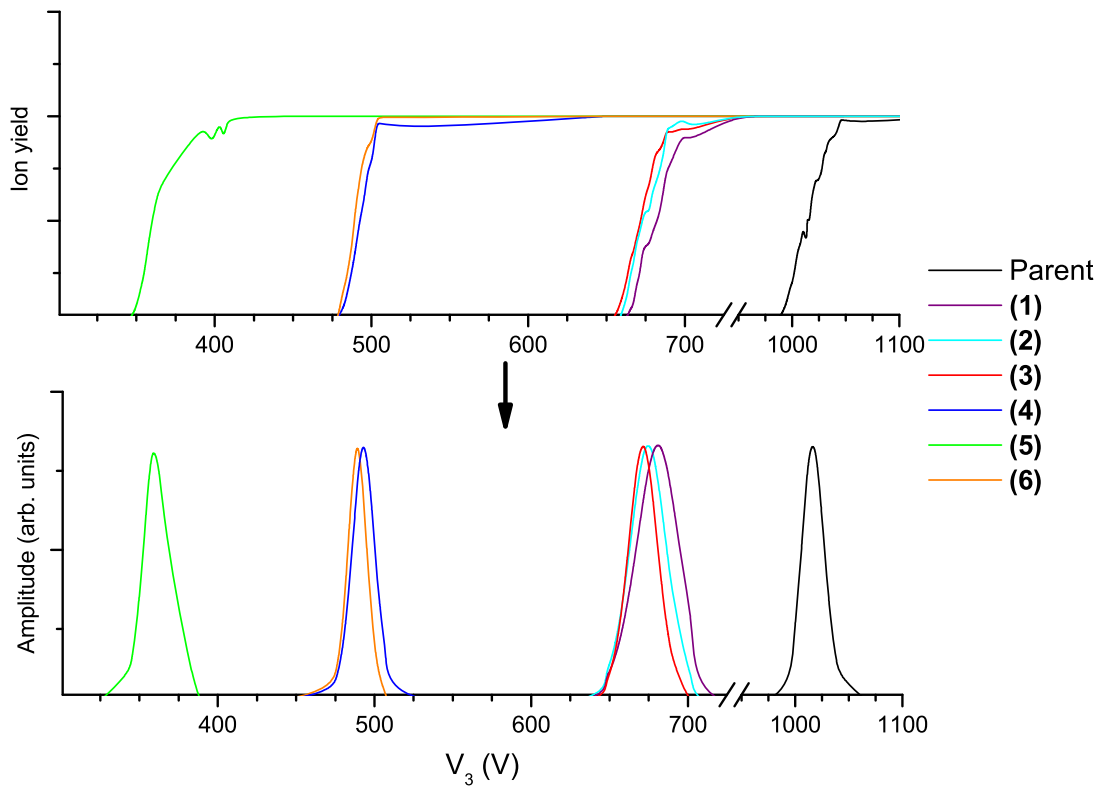

Figure 6. Taking the derivative of the experimentally-measured extinction curves (top) produces energy profiles (bottom) for each of the peaks. All of the extinction curves and kinetic energy distributions have been normalized 
metastable states of pyridine can be determined explicitly. One of these metastable states dominates in pyridine. The amount of information available by studying metastable dissociations in pyridine, coupled with the observed abundance of metastable states in similar molecules, suggests that many benzene-like systems may be good targets for further investigation of metastable states.

\section{Acknowledgments}

This material is based upon work supported by the National Science Foundation under Grant Nos. PHY-0855675 and PHY-1005071. The authors acknowledge the Max Planck Institute of Quantum Optics, and especially Dr. Hartmut Schröder, who generously lent the reflectron used in this experiment. Two authors conducted this research under research fellowships: D.B.F. (UCARE) and T.D.S. (GAANN).

\section{References}

1. Shannon, T.W., McLafferty, F.W.: Identification of gaseous organic ions by use of metastable peaks. J. Am. Chem. Soc. 88, 5021-5022 (1966)

2. Kaufmann, R., Kirsch, D., Spengler, B.: Sequencing of peptides in a time-of-flight mass spectrometer: evaluation of postsource decay following Matrix-Assisted Laser Desorption Ionization (MALDI). Int. J. Mass Spectrom. Ion Process. 131, 355-385 (1994)

3. Boesl, U., Neusser, H.J., Weinkauf, R., Schlag, E.W.: Multi-photon mass-spectrometry of metastables-Direct observation of decay in a high-resolution time-of-flight instrument. J. Phys. Chem. 86, 48574863 (1982)

4. Kuhlewind, H., Neusser, H.J., Schlag, E.W.: Metastable fragment ions in multi-photon time-of-flight mass-spectrometry-Decay channels of the benzene cation. Int. J. Mass Spectrom. Ion Process. 51, 255-265 (1983)

5. Kuhlewind, H., Neusser, H.J., Schlag, E.W.: Multiphoton metastable ion spectra and ion dissociation kinetics-Analysis of the decay channels of the aniline cation with a reflectron time-of-flight instrument. J. Chem. Phys. 82, 5452-5456 (1985)

6. Neusser, H.J.: Multiphoton mass-spectrometry and unimolecular ion decay. Int. J. Mass Spectrom. Ion Process. 79, 141-181 (1987)

7. Della-Negra, S., Le Beyec, Y.: New method for metastable ion studies with a time of flight mass spectrometer. Future applications to structure determinations. Anal. Chem. 57, 2035-2040 (1985)

8. Ioanoviciu, D., Yefchak, G.E., Enke, C.G.: Metastable peak shapes induced by internal energy-release in electrostatic mirror time-of-flight mass spectrometers. Int. J. Mass Spectrom. Ion Process. 104, 83-94 (1991)
9. Hagan, D.A., Eland, J.H.D., Lablanquie, P.: Slow dissociation reactions of singly charged ions in reflectron mass spectrometers. Int. J. Mass Spectrom. Ion Process. 127, 67-74 (1993)

10. Barofsky, D.F., Brinkmalm, G., Håkansson, P., Sundqvist, B.U.R.: Quantitative determination of kinetic energy releases from metastable decompositions of sputtered organic ions using a time-of-flight mass spectrometer with a single-stage ion mirror. Int. J. Mass Spectrom. Ion Process. 131, 283-294 (1994)

11. Cornish, T., Cotter, R.: A curved field reflectron time-of-flight massspectrometer for the simultaneous focusing of metastable product ions. Rapid Commun. Mass Spectrom. 8, 781-785 (1994)

12. Ponciano, C.R., Ávalos, F.E., Rentería, A., da Silveira, E.F.: Analysis of metastable decay by time-of-flight coincidence and kinetics energy measurements. Int. J. Mass Spectrom. 209, 197-208 (2001)

13. Ponciano, C.R., Martinez, R., da Silveira, E.F.: Fragmentation of (LiF) (n)Li + clusters in the acceleration region of TOF spectrometers. $J$. Mass Spectrom. 42, 1300-1309 (2007)

14. Cooks, R.G., Beynon, J.H.: Metastable ions and ion kinetic-energy spectrometry-Development of a new research area. J. Chem. Educ. 51, 437-443 (1974)

15. Cooks, R.G.: Metastable Ions. Elsevier Scientific, Amsterdam: NL (1973)

16. Ponciano, C.R., da Silveira, E.F.: Modeling metastable ion time-offlight peaks. J. Phys. Chem. A 106, 10139-10143 (2002)

17. Bañares, L., Baumert, T., Bergt, M., Kiefer, B., Gerber, G.: The ultrafast photodissociation of $\mathrm{Fe}(\mathrm{CO})_{5}$ in the gas phase. J. Chem. Phys. 108, 5799-5811 (1998)

18. Buzza, S.A., Wei, S., Purnell, J., Castleman Jr., A.W.: Formation and metastable decomposition of unprotonated ammonia cluster ions uupon femtosecond ionization. J. Chem. Phys. 102, 4832-4841 (1995)

19. Walker, I., Palmer, M., Hopkirk, A.: The electronic states of the azines. II. Pyridine, studied by VUV absorption, near-threshold electron energy loss spectroscopy and ab initio multi-reference configuration interaction calculations. Chem. Phys. 141, 365-378 (1990)

20. Vall-llosera, G., Coreno, M., Erman, P., Huels, M.A., Jakubowska, K., Kivimäki, A., Rachlew, E., Stankiewicz, M.: VUV photoionization of free azabenzenes: Pyridine, pyrazine, pyrimidine, pyridazine, and sTriazine. Int. J. Mass Spectrom. 275, 55-63 (2008)

21. de Boer, M.P., Muller, H.G.: Observation of large populations in excited states after short-pulse multiphoton Ionization. Phys. Rev. Lett. 68, 2747-2750 (1992)

22. Bordyug, N.V., Krainov, V.P.: Dynamic resonances in ultra-short laser pulses. Laser Phys. Lett. 4, 418-420 (2007)

23. Scarborough, T.D., Foote, D.B., Uiterwaal, C.J.G.J.: Ultrafast resonance-enhanced multiphoton ionization in the azabenzenes: pyridine, pyridazine, pyrimidine, and pyrazine. J. Chem. Phys. in press. doi:10.1063/1.3682541. (2012)

24. Strohaber, J., Uiterwaal, C.J.G.J.: In situ measurement of threedimensional ion densities in focused femtosecond pulses. Phys. Rev. Lett. 100, 023002 (2008)

25. Lin, M., Dyakov, Y.A., Tseng, C., Mebel, A.M., Lin, S.H., Lee, Y.T., Ni, C.: Photodissociation dynamics of pyridine. J. Chem. Phys. 123, 054309 (2005)

26. Jung, S.H., Yim, M.K., Choe, J.C.: Loss of HCN from the pyrazine molecular ion: A theoretical study. Bull. Korean Chem. Soc. 32, 23012305 (2011) 